ITC $4 / 50$

Information Technology and Control

Vol. 50/ No. 4/2021

pp. 736-751

DOI 10.5755/j01.itc.50.4.28104
Comparison of Feedback Control Strategies for Operation of Granulation Loops

\begin{tabular}{|c|c|}
\hline Received 2020/12/02 & Accepted after revision 2021/11/05 \\
\hline Cross ef http://dx.doi.org/10.5755/j01.itc.50.4.28104 \\
\hline
\end{tabular}

HOW TO CITE: Vesjolaja, L., Glemmestad, B., Lie, B. (2021). Comparison of Feedback Control Strategies for Operation of Granulation Loops. Information Technology and Control, 50(4), 736-751. https://doi.org/10.5755/j01.itc.50.4.28104

\title{
Comparison of Feedback Control Strategies for Operation of Granulation Loops
}

\section{Ludmila Vesjolaja}

Department of Electrical Engineering, IT and Cybernetics, University of South-Eastern Norway; e-mail:ludmila.vesjolaja@usn.no

\section{Bjørn Glemmestad}

Digital Production Department, Yara International ASA, Norway; e-mail: bjorn.glemmestad@yara.com

\section{Bernt Lie}

Department of Electrical Engineering, IT and Cybernetics, University of South-Eastern Norway;

e-mail: bernt.lie@usn.no

Corresponding author:ludmila.vesjolaja@usn.no

Granulation is a particle enlargement process during which fine particles or atomizable liquids are converted into granules via a series of complex granulation mechanisms. In this paper, two feedback control strategies are implemented to make granulation loop processes more steady to operate, i.e., to suppress oscillatory behavior in the produced granule sizes. In the first control strategy, a classical proportional-integral (PI) controller is used, while in the second, a double-loop control strategy is used to control the median diameter of the granules leaving the granulator. The simulation results showed that using the proposed control design for the granulation loop can eliminate the oscillatory behaviour in the produced granule median diameter and make granulation loop processes more steady to operate. A comparison between the two proposed control strategies showed that it is preferable to use the double-loop control strategy.

KEYWORDS: granulation loop, oscillatory behavior, dynamic modeling, population balance, automatic control, PID controller. 


\section{Introduction}

Granulation is a particle design technique during which fine particles are converted into larger particles called granules. As a result of granulation process, granules with the desired properties are produced. The desired granule properties depend on the product quality requirements. Some of the common granule properties of interest are particle size, moisture content, porosity, compressibility and etc. Granule formation and their properties also depends on granulation mechanisms. According to Iveson et al. [8], there are three main granulation mechanisms: (i) particle nucleation that is the initial stage of particle formation, (ii) particle growth that can occur due to various mechanisms such as particle layering and and particle agglomeration, and (iii) particle breakage, e.g., particle surface attrition due to particle collision [8], [3].

This paper is focused on the granulation process used in fertilizer industry. A rotary drum granulator is used to produce particles with the desired particle properties. Here, the particle property of interest is the particle size distribution (PSD). Granule growth inside the drum granulator is assumed due to (i) particle layering, and (ii) binary particle agglomeration as shown in Figure 1. Here, particle layering is considered as a continuous process during which particle growth occurs by successive coating of the slurry onto a particle. Binary particle agglomeration is considered as a process that occurs due to successful collision of two particles, resulting in the formation of a larger, composite particle [8], [12], [31], [30].

In fertilizer industry, there are operational challenges in the granulation processes that are of interest in terms of process control view. Control of granulation processes is needed since (i) granulation processes are operated below design capacity, and (ii) oscillatory behavior may occur in the produced granule size and in the granule flow rates. This paper focuses on damping and elimination of oscillatory behavior seen on granulation loop plants.

Oscillatory behavior is observed on different, with regard to the granulator type, granulation loop plants. For example, studies on oscillatory behaviour seen in fluidized bed granulators are given in [17], [23]. Numerous studies are focused on control of fluidized bed granulators, including [1], [2], [4], [19], [20], [21]. The recent studies regarding stabilization of the continuous granulation loop process in the fluidized bed are reported in [17], [18]. Similarly to fluidized bed granulators, granulation loop processes using drum granulators also show oscillatory behavior of particle sizes and particle flow rates [29], [30]. Studies regarding the control of the granulation processes where the rotary drums are used as the granulators are given in [5], [24], [29], [33], [32].

A mathematical model of the granulation process is needed to study the possibilities for eliminating the oscillatory behaviour seen on industrial scale granulation loop plants. There are several approaches to modeling granulation processes. Typically modeling approahes are divided into (i) black box models also referred as empirical models that are based on actual plant data, and (ii) grey-box models called also mechanistic models that are based on conservation

Figure 1

Particle growth due to particle layering and particle agglomeration inside the drum granulator [31]

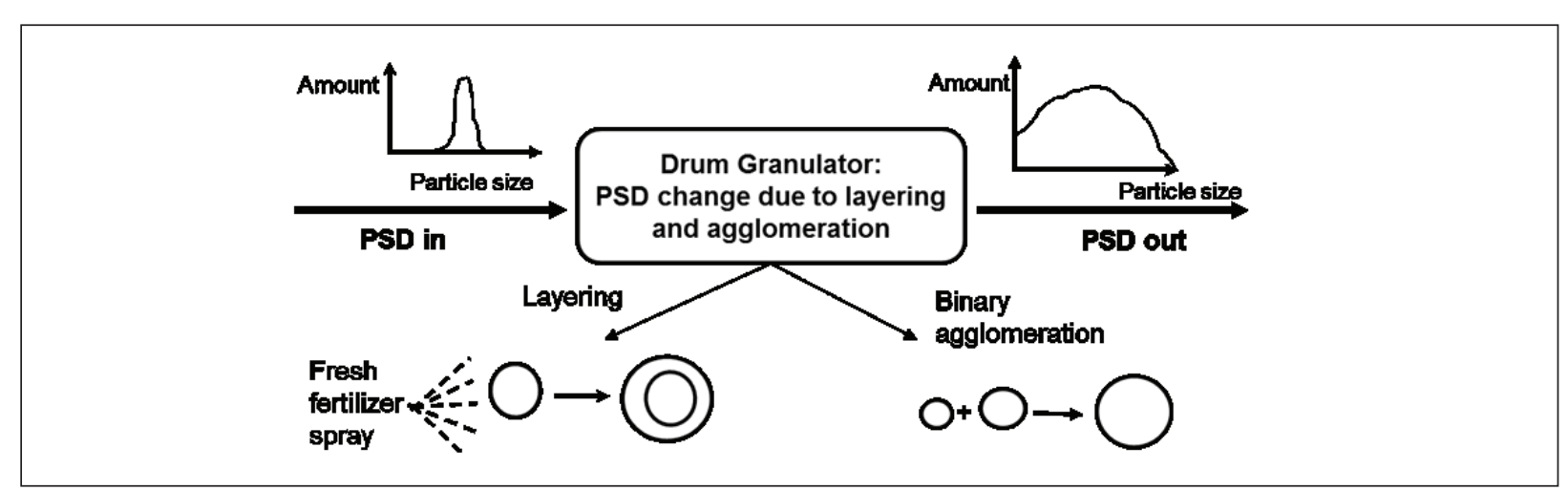


principles. Mathematical modeling of the granulation processes for control studies is commonly performed using the population balance (PB) principle [26]. In [3], [18], [23], [31], [30], [7], [25], [28], [33], [32] mechanistic models using the PB principles are developed. Generic drum granulation models are presented in [24], [25], [28]. In this paper, a mechanistic model using the $\mathrm{PB}$ principles is used to model the granulation process.

Even though numerous research works have been performed in studying granulation processes, the control of the granulation loop process still remains a challenge. The motivation of this research is to design proper control strategies to make production of granulation loop processes more steady to operate, i.e., to eliminate the oscillatory behavior observed in the produced particle sizes.

This paper is the extension of our previous studies reported in. In this paper, the authors extend the previous study [29] by comparing two feedback control strategies with the objective to eliminate the oscillatory behaviour in the the produced particle sizes. Thus, the main contribution of this paper is the comparison of the double-loop control strategy [29] with the classical PI controller. Another contribution of this paper is the analysis of the system dynamics using a bifurcation analysis.
The paper is organized as follows: In Section 2, the description and the model of the granulation loop are given. Open loop simulations of the dynamic model are performed in Section 3. The design of the control system for the granulation loop process is given in Section 4.1. In Section 4.2, a classical PI-controller is applied to the granulation loop process. A description of a double-loop control strategy is provided in Section 4.3. Simulation results and discussion are given in Section 5, while conclusions are drawn in Section 6. Descriptions of the symbols used in the paper are listed in Appendix 1.

\section{Granulation Loop}

\subsection{Process Description}

The granulation loop with the recycling studied in this paper is shown in Figure 2. The main granulation loop units are: (i) rotary drum granulator, (ii) double-deck screen (particle classifier), and (iii) roll crusher. The rotary drum is used to produce solid particles (granules) by spraying a slurry melt on recycled particles. In the granulation drum, particles grow in their sizes due to various granulation mechanisms. In this paper, the particle growth is assumed to occur due to both the particle layering and the binary particle agglomeration [8], [12], [31], [30].

\section{Figure 2}

Schematic diagram of the rotary drum granulation loop used to model granulation process in fertilizer industry [30]

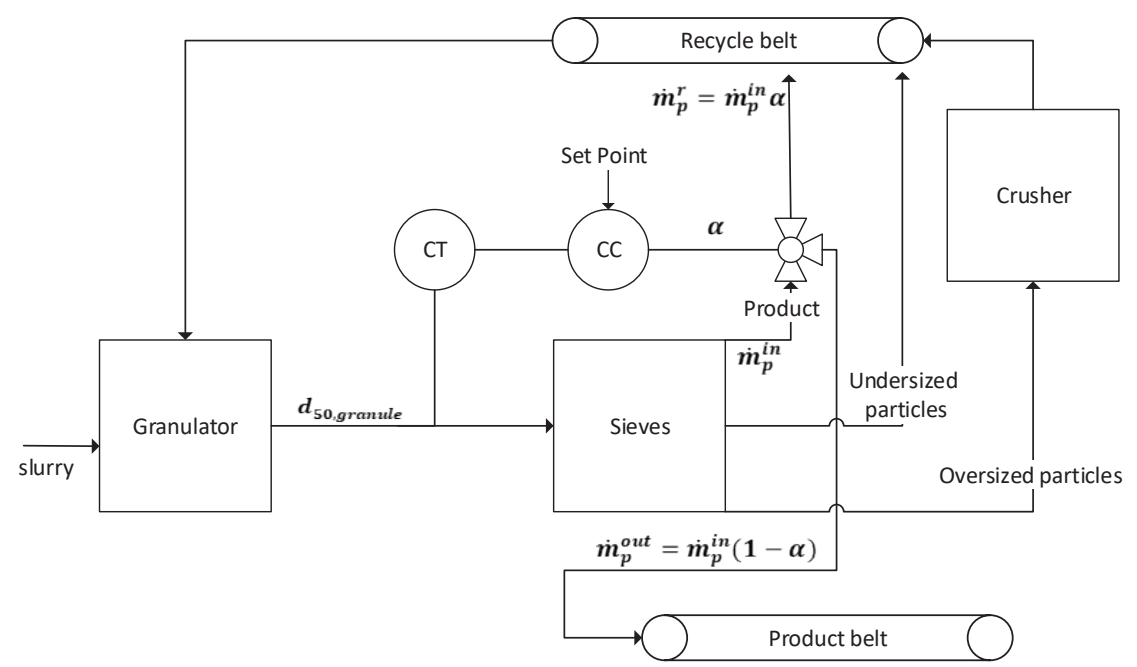


Together with the granulator, the granulation loop also consists of a product classification step (double-deck screens). Granules that remain lying on the upper screen (over-sized particles) are sent to the crusher. The crushed granules act as a seed to the granulator, and are recycled back to the granulator. Granules that pass through the upper screen but remain lying on the lower screen are the product-sized particles that are collected for further use. Granules that are too small to remain lying on the upper nor on the lower screen are the under-sized particles that are recycled back to the granulator together with the crushed over-sized particles. Thus, the recycle feed contains both the crushed over-sized particles and the under-sized particles.

\subsection{Mathematical Model}

The overall flow scheme for the drum granulation loop is given in Figure 3 (Symbol descriptions are given in Appendix 1). A continuous granulation process in a rotary drum was modeled using a mass based population balance equation (PBE). Here, the developed models are 2-dimensional models with respect to particle size and position in the granulator. The resulting PBE is partial differential equation (PDE) that is discretized in terms of its internal coordinate (particle diameter) and external coordinate (axial position in the granulator).

The mass based PBE with particle diameter $(d)$ as the internal coordinate, spatial variation $(z)$ as the external coordinate, and time coordinate $(t)$ is

$$
\begin{aligned}
& \frac{\partial m(d, z, t)}{\partial t}=-d^{3} \frac{\partial}{\partial d}\left[G \frac{m(d, z, t)}{d^{3}}\right]+B(d, z, t) \\
& -D(d, z, t)-\frac{\partial}{\partial z}[v \cdot m(d, z, t)],
\end{aligned}
$$

where $m(d, z, t)$ is the mass density function [29], [30], [31]. Equation (1) is formed by assuming the two granulation mechanisms, particle growth due to layering and particle agglomeration. Equation (1) is derived from a number based PBE as in [26], [31]. Derivation of Equation (1) and mathematical expressions for the $G, B, D$ terms in Equation (1) are reported in detail in [31], [30]. Mathematical models of the screens and the crusher (Figure 3) are given in Table 2.3.

\subsection{Model Solution}

The solution to PBEs is found by discretizing the PDE (Equation (1)) into a system of ordinary differential equations (ODEs). The PDEs for PBE are discretized in terms of the internal coordinate, i.e., particle diameter, and also in terms of the external coordinate, i.e., the position in the drum granulator.

The two granulation mechanisms, particle layering and particle agglomeration, require different discretization algorithms. In this paper, the layering term in Equation (1) is discretized using a finite volume scheme extended by a Koren flux limiter function (KFL) [9], [30]. The agglomeration term in Equation (1) is discretized using

Figure 3

Simplified flow diagram of the drum granulation loop used for modeling the granulation loop [30]

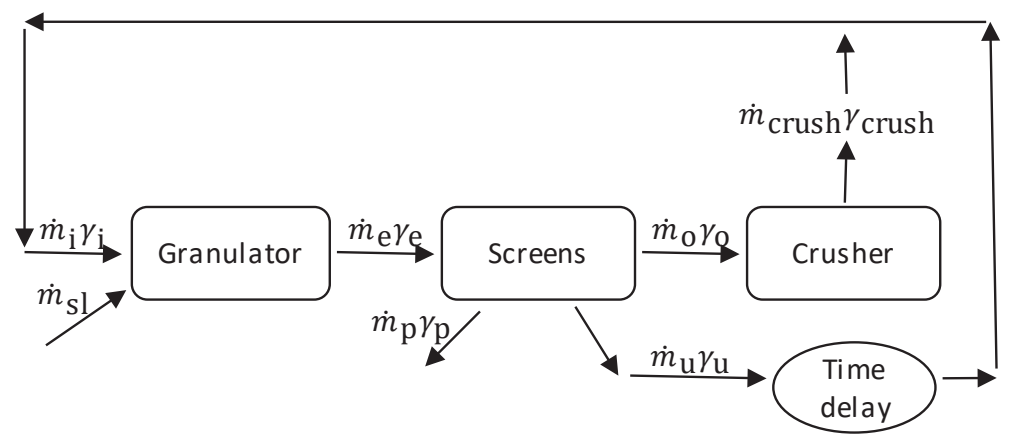


Table 1

Model expressions for the screens and the crusher in Figure 3

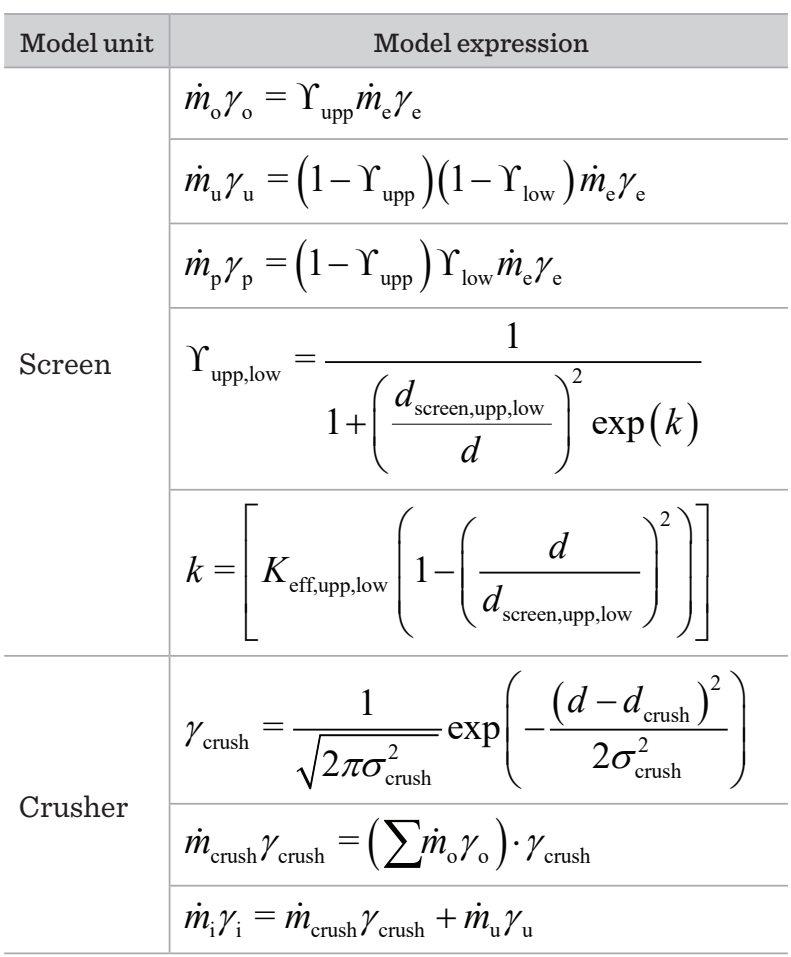

a cell average scheme described in [10], [11], and applied to a mass-based PBE in [30], [31].

Model expressions for the screens and the crusher in Figure 3.

If $N_{c}$ is the total number of particle size classes (cells), the discretized PBE in terms of particle size can be written as,

$$
\begin{aligned}
\frac{d M_{i}}{d t} & =d^{3}\left[G m\left(t, d_{i-\frac{1}{2}}\right)-G m\left(t, d_{i+\frac{1}{2}}\right)\right] \\
& +d_{i}^{3}\left[B_{i-1} \lambda_{i}^{-}\left(\bar{d}_{i-1}\right) H\left(\bar{d}_{i-1}-d_{i-1}\right)\right. \\
& +B_{i} \lambda_{i}^{-}\left(\bar{d}_{i}\right) H\left(d_{i}-\bar{d}_{i}\right) \\
& +B_{i} \lambda_{i}^{+}\left(\bar{d}_{i}\right) H\left(\bar{d}_{i}-d_{i}\right) \\
& \left.+B_{i+1} \lambda_{i}^{+}\left(\bar{d}_{i+1}\right) H\left(d_{i+1}-\bar{d}_{i+1}\right)\right] \\
& -M_{i} \sum_{k=1}^{N_{c}} \beta_{i k} \frac{M_{k}}{d_{k}^{3}}+\frac{\partial}{\partial z}\left(v \cdot m_{i, z}\right),
\end{aligned}
$$

where

$$
\begin{aligned}
m\left(z, t, d_{i-\frac{1}{2}}\right) \approx & \frac{1}{\Delta d}\left\{\frac{M_{i-1}}{d_{i-1}}+\frac{1}{2} \phi\left(\theta_{i-\frac{1}{2}}\right)\right. \\
& \left.\times\left(\frac{M_{i-1}}{d_{i-1}^{3}}-\frac{M_{i-2}}{d_{i-2}^{3}}\right)\right\}, \\
m\left(z, t, d_{i+\frac{1}{2}}\right) \approx & \frac{1}{\Delta d}\left\{\frac{M_{i}}{d_{i}}+\frac{1}{2} \phi\left(\theta_{i+\frac{1}{2}}\right)\right. \\
& \left.\times\left(\frac{M_{i}}{d_{i}^{3}}-\frac{M_{i-1}}{d_{i-1}^{3}}\right)\right\},
\end{aligned}
$$

with

$$
\theta_{i-\frac{1}{2}}=\frac{\frac{M_{i}}{d_{i}^{3}}-\frac{M_{i-1}}{d_{i-1}^{3}}+\varepsilon}{\frac{M_{i-1}}{d_{i-1}^{3}}-\frac{M_{i-2}}{d_{i-2}^{3}}+\varepsilon},
$$

and

$$
\theta_{i+\frac{1}{2}}=\frac{\frac{M_{i+1}}{d_{i+1}^{3}}-\frac{M_{i}}{d_{i}^{3}}+\varepsilon}{\frac{M_{i}}{d_{i}^{3}}-\frac{M_{i-1}}{d_{i-1}^{3}}+\varepsilon} .
$$

The birth $B_{i}$ of the particles are given as

$$
B_{i}=\frac{1}{2} \sum_{j=1}^{i} \sum_{k=1}^{i} \beta_{j k} \frac{M_{j}}{d_{j}^{3}} \frac{M_{k}}{d_{k}^{3}},
$$

and the average diameter of all the new-born particles in the $i^{\text {th }}$ class is

$$
\bar{d}_{i}=\left[\frac{\sum_{j=1}^{i} \sum_{k=1}^{i} \beta_{j k} \frac{M_{j}}{d_{j}^{3}} \frac{M_{k}}{d_{k}^{3}}\left(d_{j}^{3}+d_{k}^{3}\right)}{\sum_{j=1}^{i} \sum_{k=1}^{i} \beta_{j k} \frac{M_{j}}{d_{j}^{3}} \frac{M_{k}}{d_{k}^{3}}}\right]^{\frac{1}{3}} .
$$

Agglomeration kernels $\left(\beta_{j k}\right.$ and $\beta_{i k}$ ), the dimensionless term $\lambda_{i}^{ \pm}(d)$ and the Heaviside step function $H$ in Eq. (2) are defined in [30], [31]. 
Assuming constant particle velocity inside the granulator space, the external coordinate discretization is

$$
v \frac{\partial}{\partial z}\left(m_{i, z}\right)=v\left[m_{i, z-\frac{1}{2}}-m_{i, z+\frac{1}{2}}\right] .
$$

The approximation of the terms $m_{i, z \pm \frac{1}{2}}$ is performed using the KFL scheme similarly to the layering term approximation. The resulting expressions are given in detail in[30].

\section{System Dynamics}

\subsection{Simulation Setup}

Dynamic simulations are performed using MATLAB and Simulink [14]. Simulation setup is the same as given in [29]. Simulation parameters are listed in Table 2.

Table 2

Simulation parameters [29]

\begin{tabular}{l|l}
\hline \multicolumn{1}{c|}{ Parameter } & \multicolumn{1}{c}{ Value } \\
\hline Range of $d[\mathrm{~mm}]$ & $0-8$ \\
\hline Number of particle classes & 80 \\
\hline Grid type & linear \\
\hline Length of granulator $[\mathrm{m}]$ & 10 \\
\hline Number of compartments & 3 \\
\hline$\rho$ [kg $\left.\cdot \mathrm{m}^{-3}\right]$ & 1300 \\
\hline$\beta_{0}\left[\mathrm{~s}^{-1}\right]$ & $1.0 \cdot 10^{-13}$ \\
\hline$\dot{m}_{\text {sl,i }}\left[\mathrm{kg} \cdot \mathrm{h}^{-1}\right]$ & 1000 \\
\hline$d_{\text {screen,upp }[\mathrm{mm}]}$ & 3.3 \\
\hline$d_{\text {screen,low }}[\mathrm{mm}]$ & 1.9 \\
\hline$K_{\text {eff,upp }}$ & 45 \\
\hline$K_{\text {eff,low }}$ & 45 \\
\hline$d_{\text {crush }}[\mathrm{mm}]$ & $2.0-1.0$ \\
\hline$\sigma_{\text {crush }}$ & 0.25 \\
\hline$T_{\mathrm{R}}[\mathrm{s}]$ & 600 \\
\hline Transport delay $[\mathrm{s}]$ & 600 \\
\hline & \\
\hline & \\
\hline
\end{tabular}

\subsection{Oscillatory Behavior}

The dynamic model developed for the rotary drum granulation loop is able to capture the main dynamics of the granulation process. Through dynamic simulations, the model was found to reproduce the oscillatory behaviour typically seen in granulation loops. In particular, the developed model is able to reproduce oscillatory behaviour in the particle size distribution, measured as the particle size $d_{50}$ diameter (median diameter that corresponds to the intercept for $50 \%$ of cumulative mass), Figure 4.

Figure 4

Simulated PSD of the effluent from the granulator

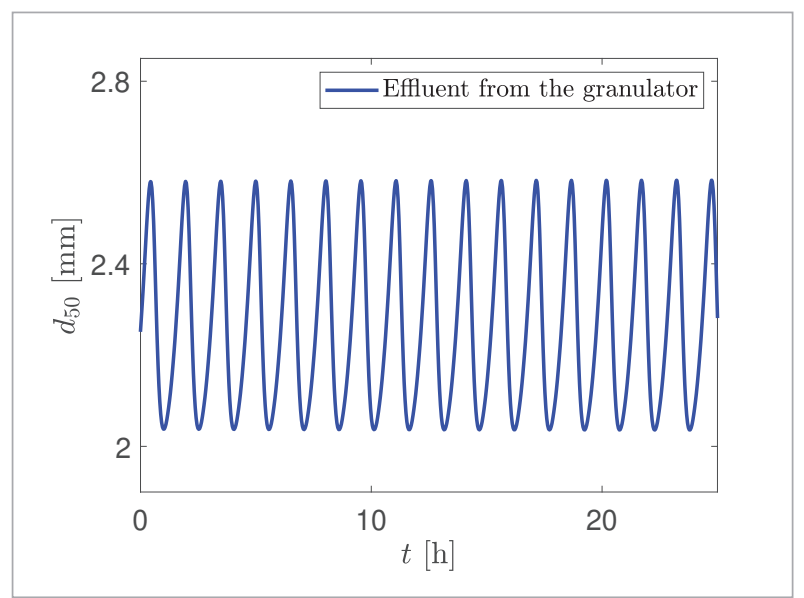

Similarly to the simulation results, oscillatory behaviour is also observed in the industrial-scale plants as shown in Figure 5. Oscillatory behaviour is observed not only in the granule $d_{50}$ but also in the product quantity (mass flow rates). Illustrations showing oscillatory behavior in product quantity are given in [30] and not illustrated in this paper for the sake of brevity.

\section{Figure 5}

Oscillatory behavior in PSD of an industrial granulation loop process [29]

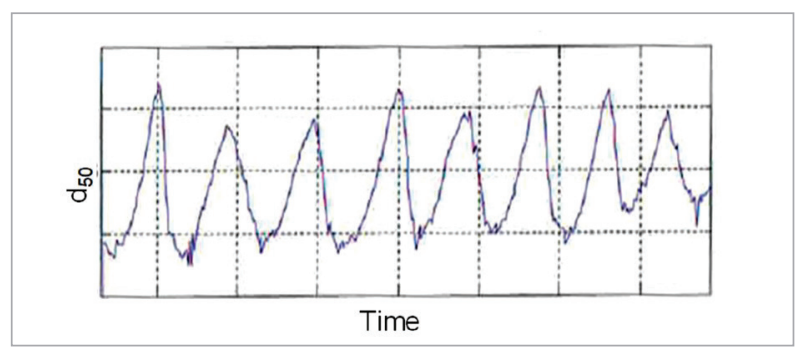


Further insight into the system dynamics of the granulation loop process is obtained from a bifurcation analysis. Here, a simple bifurcation analysis based on model simulations was performed. For this, the system was simulated until stationary conditions were reached, and the upper and the lower $d_{50}$ value was registered when the system oscillated. In these simulations, the crusher gap spacing $d_{\text {crush }}$ was chosen as a bifurcation parameter to analyse the change in the behaviour of the PSD, being measured as $d_{50}$, in the effluent from the granulator. The simulations were performed with the different values of $d_{\text {crush }}$, from 2 $\mathrm{mm}$ to $1 \mathrm{~mm}$ with an interval of $0.1 \mathrm{~mm}$. Stable and unstable points (oscillatory behavior) of $d_{\text {crush }}$ were determined using the obtained amplitude of stationary solutions at different $d_{\text {crush }}$ values.

Simulation results of the bifurcation analysis are illustrated in Figure 6. In Figure 6, the minimum and the maximum $d_{50}$ value of the oscillations at a certain $d_{\text {crush }}$ is depicted. These simulation results showed that at larger crusher gap spacings, a stable granulation process is observed $\left(d_{\text {crush }}<1.5 \mathrm{~mm}\right)$. However, as the crusher gap spacing is reduced, the granulation loop starts to show oscillatory behavior, and at a certain crusher gap spacing $\left(d_{\text {crush }}<1.4 \mathrm{~mm}\right.$ ), sustained periodic oscillations occur. The probable reason of the oscillatory behavior of the granulation process is described in [30].

\section{Figure 6}

Bifurcation plot with the crusher gap spacing $d_{\text {crush }}$ as bifurcation variable

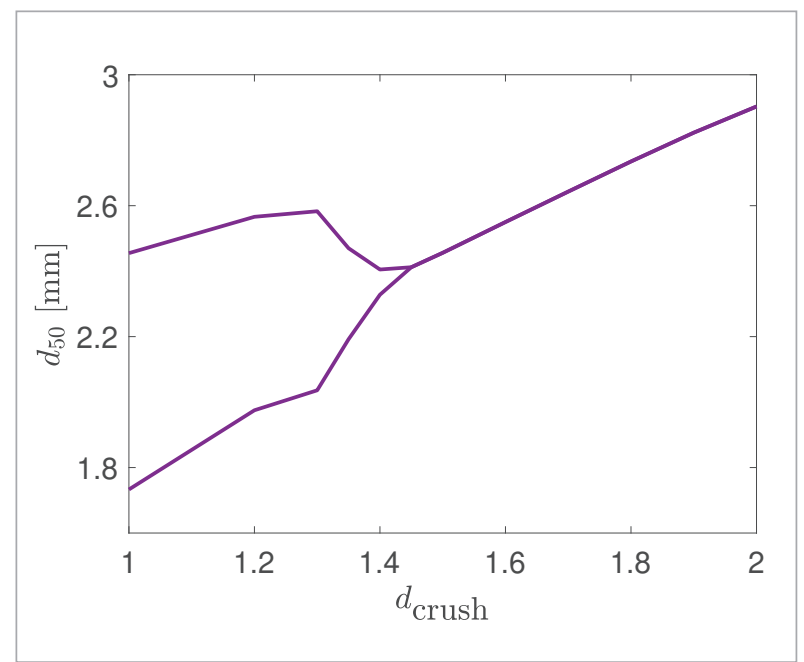

\section{Control of the Granulation Loop}

\subsection{Design of the Control System}

In this paper, the stability of the $d_{50}$ diameter is performed by implementing a controller having a 3-way valve opening $\alpha$ as the control input. When the valve $\alpha$ is opened, i.e., $0<\alpha \leq 1$, some fraction of the product-sized particle mass flow rate is added to the recycle flow. The control output is the particle $d_{50}$ diameter in the effluent, i.e., on the elevator coming from the granulator before the granules are sieved. Details regarding the design of the control system are given in [29].

\subsection{Classical PI-Controller}

Here, a classical proportional-integral PI-controller was used to eliminate the oscillatory behavior of the process. The schematic diagram of the PI-controller for the granulation loop process (oscillatory process) is illustrated in Figure 7.

\section{Figure 7}

Classical PI-controller control strategy for granulation loop process. $u=\alpha-$ control signal (valve opening); $y-$ controlled variable ( $d_{50}$ of the effluent); r - reference point

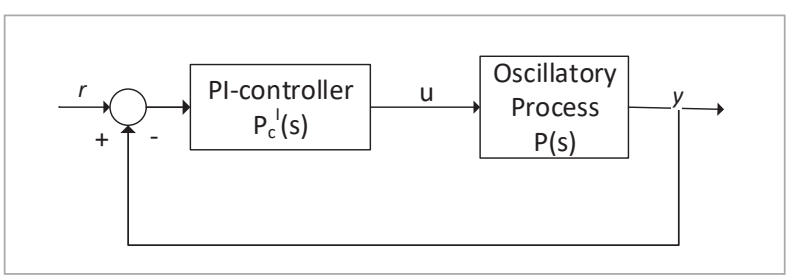

The granulation plant can be approximated as an oscillatory process having a transfer function

$$
P(s)=\frac{K_{\mathrm{p}} e^{-\tau s}}{1+\frac{2 \zeta s}{\omega_{0}}+\left(\frac{s}{\omega_{0}}\right)^{2}}
$$

where $P(s)$ is the transfer function of the underdamped (oscillatory) process, $K_{\mathrm{p}}$ is the process gain, $\tau$ is the process time delay, $\omega_{0}$ is the natural frequency and $\zeta$ is the damping factor.

Process parameters $K_{\mathrm{p}}, \tau$, $\zeta$, and $\omega_{0}$ were found using a simulated step-response of the granulation process. For this, a step-change of the valve opening (ratio of the product-sized particles to be recycled) is given 
to the system. An example of such a step-response is shown in Figure 8. Expressions for calculating process parameters are summarized in Table 1.

\section{Figure 8}

Illustrative example of a step-response: A change in $d_{50}$ of the effluent from the granulator as a response to a step change in the valve opening $\alpha ; T_{0}$-period of the oscillation and $A_{1}$, $A_{2}$-amplitudes of the first two overshoots of the 2 nd order response

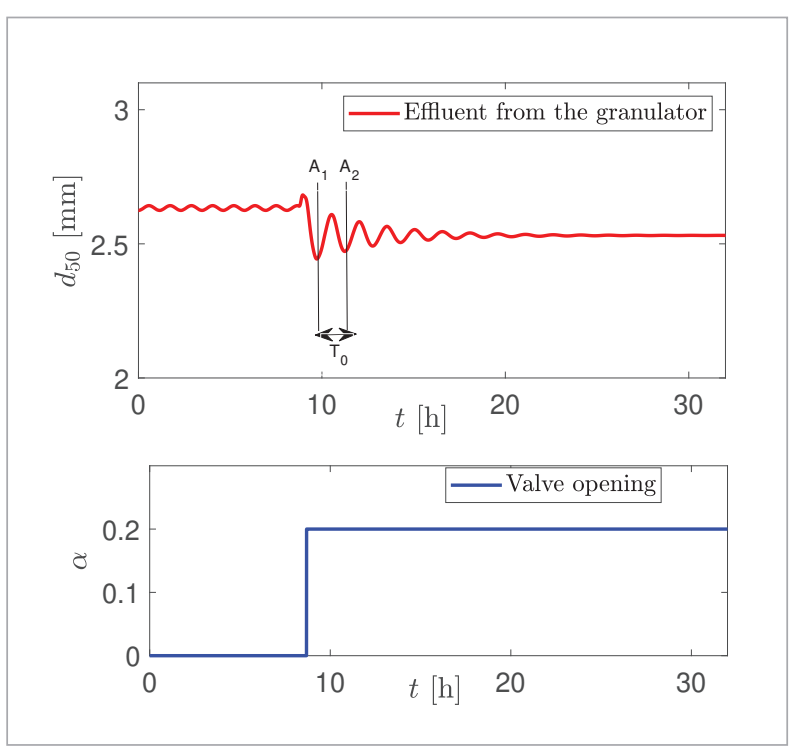

Table 3

Model parameter identification

\begin{tabular}{c|c}
\hline Parameter & Expression \\
\hline$\zeta$ & $\frac{1}{\sqrt{1+\left(\frac{2 \pi}{\sigma}\right)^{2}}}$ \\
\hline$\omega_{0}$ & $\frac{2 \pi}{T_{0}}$ \\
\hline$\sigma$ & $\ln \left(\frac{A_{1}}{A_{2}}\right)$
\end{tabular}

The PI controller equation in frequency domain is written as,

$$
P_{\mathrm{c}}^{\mathrm{I}}(s)=K_{\mathrm{c}}^{\mathrm{I}}\left(1+\frac{1}{T_{\mathrm{i}}^{\mathrm{I}} s}\right),
$$

Table 4

Expressions for designing the classical PI controller $P_{\mathrm{c}}^{\mathrm{I}}(s)$

\begin{tabular}{c|c}
\hline Parameter & Expression \\
\hline$K_{\mathrm{c}}^{\mathrm{I}}$ & $\frac{2\left(\frac{1}{\omega_{0}} \zeta\right)}{k\left(T_{\mathrm{c}}^{\mathrm{I}}+\tau\right)}$ \\
\hline$T_{\mathrm{i}}^{\mathrm{I}}$ & $2\left(\frac{1}{\omega_{0}} \zeta\right)$ \\
\hline$T_{\mathrm{c}}^{\mathrm{I}}$ & $-\tau<T_{\mathrm{c}}^{\mathrm{I}}<\infty$ \\
\hline
\end{tabular}

where $K_{\mathrm{c}}^{\mathrm{I}}$ is the proportional gain of the PI controller and $T_{\mathrm{i}}^{\mathrm{I}}$ is the integral time constant of the PI controller. The PI controller tuning parameters, $K_{\mathrm{c}}^{\mathrm{I}}$ and $T_{\mathrm{i}}^{\mathrm{I}}$, were found using Skogestad's tuning rules for oscillatory processes [13] that are given in Table 2 .

\subsection{Double-Loop Control Strategy}

In this section, a double-loop control strategy for oscillatory systems [16], [22], [29], is applied to control the $d_{50}$ diameter in the effluent from the granulator. The double-loop control strategy consists of two controllers, an inner controller and an outer controller. The proposed double-loop control strategy is illustrated in Figure 9.

\section{Figure 9}

Representation of the double-loop control strategy as: (a) double-loop control algorithm, (b) single-loop control algorithm [16]; $u=\alpha$ - control signal (valve opening), $y=d_{50}-$ controlled variable ( $d_{50}$ of the effluent), $\mathrm{r}$ - reference point

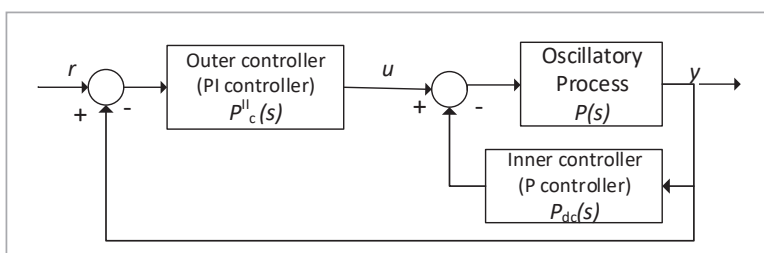

(a)

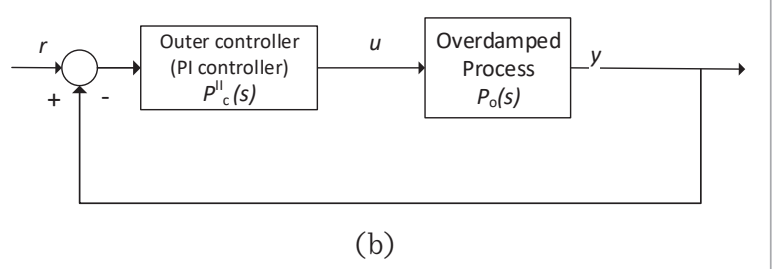


The inner controller $\left(P_{\mathrm{dc}}(s)\right.$ in Figure 9$)$ is used to convert the underdamped process $(P(s)$ in Figure 9$)$ to an overdamped process $\left(P_{0}(s)\right.$ in Figure 9$)$. The outer controller $\left(P_{\mathrm{c}}^{\mathrm{II}}(s)\right.$ in Figure 9$)$, which is the main controller, is used to achieve specified performance. The double-loop control strategy resembles the cascade control structure. However, the main differences between the double-loop control structure and the conventional cascade control structure are:

\section{Table 5}

Expressions for designing the double-loop control strategy $P_{\mathrm{c}}^{\mathrm{II}}(s)$

\begin{tabular}{|c|c|}
\hline \multirow{6}{*}{ 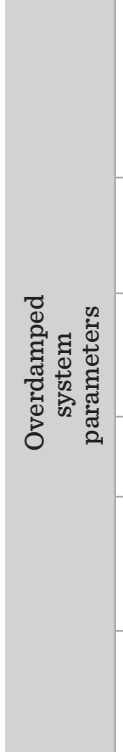 } & $K_{\mathrm{c}}^{\mathrm{i}}=2 \cdot \frac{\left(\zeta_{\mathrm{i}}^{2}+\zeta \tau \omega_{0}\right) \pm \sqrt{\left(\zeta_{\mathrm{i}}^{2}+\zeta \tau \omega_{0}\right)^{2}+\tau^{2} \omega_{0}^{2}\left(\zeta_{\mathrm{i}}^{2}-\zeta^{2}\right)}}{\tau^{2} w_{0}^{2} K_{\mathrm{p}}}$ \\
\hline & $\omega_{\mathrm{i}}=\omega_{0} \sqrt{1+K_{\mathrm{c}}^{\mathrm{i}} K_{\mathrm{p}}}$ \\
\hline & $K_{\mathrm{p}}^{\mathrm{i}}=\frac{K_{\mathrm{p}}}{1+K_{\mathrm{c}}^{\mathrm{i}} K_{\mathrm{p}}}$ \\
\hline & $\zeta_{\mathrm{i}}>1$ \\
\hline & $T_{1}=\frac{\zeta_{\mathrm{i}}+\sqrt{\zeta_{\mathrm{i}}^{2}-1}}{\omega_{\mathrm{i}}}$ \\
\hline & $T_{2}=\frac{\zeta_{\mathrm{i}}-\sqrt{\zeta_{\mathrm{i}}^{2}-1}}{\omega_{\mathrm{i}}}$ \\
\hline 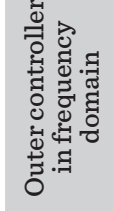 & $P_{\mathrm{c}}^{\mathrm{II}}(s)=K_{\mathrm{c}}^{\mathrm{II}}\left(1+\frac{1}{T_{\mathrm{i}}^{\mathrm{II}} s}\right)$ \\
\hline \multirow{5}{*}{ 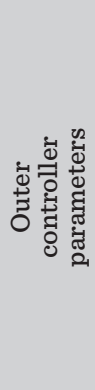 } & $K_{\mathrm{c}}^{\mathrm{II}}=\frac{T_{\text {eff }}}{K_{\mathrm{p}}^{\mathrm{i}}\left(T_{\mathrm{c}}^{\mathrm{II}}+\tau_{\mathrm{eff}}\right)}$ \\
\hline & $T_{\mathrm{i}}^{\mathrm{II}}=\min \left(T_{\mathrm{eff}}, 4\left(T_{\mathrm{c}}^{\mathrm{II}}+\tau_{\mathrm{eff}}\right)\right)$ \\
\hline & $-\tau_{\mathrm{eff}}<T_{\mathrm{c}}^{\mathrm{II}}<\infty$ \\
\hline & $\tau_{\text {eff }}=\tau+0.5 T_{2}$ \\
\hline & $T_{\text {eff }}=T_{1}+0.5 T_{2}$ \\
\hline
\end{tabular}

- With the double-loop control structure, the same measurement, $y$, is used for both the inner controller and the outer controller (Figure 9). In the conventional cascade control structure, usually the measurement used for the inner loop controller and the measurement used for the outer loop controller are different. The measurement for the outer control loop, for the conventional cascade control structure, is the controlled variable of the system that needs to be tracked to a given set (reference) point.

- With the double-loop control structure, the inner and the outer control loops are usually not categorized into slow or fast controller loops as is usually done in the conventional cascade control structure.

The combined transfer function from $u$ to $y$ in Figure 9 is

$$
P_{\mathrm{o}}(s)=\frac{P(s)}{1+P(s) \cdot P_{\mathrm{dc}}(s)}=\frac{P(s)}{1+P(s) \cdot K_{\mathrm{c}}^{\mathrm{i}}},
$$

where $P_{\mathrm{o}}(s)$ is the transfer function of the overdamped system and $P_{\mathrm{dc}}(s)$ is the transfer function of the inner (damping) controller. Here, the damping controller is a P-controller, i.e., $P_{\mathrm{dc}}(s)=K_{\mathrm{c}}^{\mathrm{i}}$.

Expressions for finding process parameters of the overdamped system are summarized in Table 9 . The expressions listed in Table 9 are derived by using an approximation of the time delay as $e^{-\tau s} \approx 1-\tau s$. For the outer controller, the PI controller was used. The outer controller was used to track a reference point. Tuning of the outer controller was performed using SIMC PI tuning rules given in [27] and applied to the granulation control system in [29].

Expressions used to find the PI controller tuning parameters, overdamped process parameters and outer controller parameters, for the double-loop control strategy are summarized in Table 9. In overdamped systems, the damping factor is greater than 1. In this study, the value for the inner loop damping factor is set to $\zeta_{\mathrm{i}}=1.5$. Underdamped process parameters, $K_{\mathrm{p}}, \tau$, and $\omega_{0}$, are calculated using expressions given in Table 1, i.e., were found using a simulated step-response of the granulation process shown in Figure 8. 


\section{Simulation Results}

Feedback control strategies are applied to make granulation loops more steady in operation. The closed loop simulations were performed using the design of the control system of the granulation loop process described in Section 4.1. The controller is implemented to suppress the oscillatory behavior of the process parameters as discussed in Section 3. In particular, the controller is designed to control the PSD, measured as $d_{50}$, of the particles leaving the granulator. Thus, the control output is the $d_{50}$ of the particles leaving the granulator (effluent from the granulator). Stabilization of the oscillatory behavior is performed by recycling some of the product-sized particles back to the granulator. Thus, the control input is the valve opening $\alpha$ that controls the recycled product flow.

Two control strategies are used to control the $d_{50}$ diameter: (i) a classical PI-controller and (ii) a double-loop control strategy. First, the closed simulations were performed using the classical PI-controller. The PI-controller was tuned using SIMC tuning rules for the oscillatory systems. Then fine tuning was done to achieve better performance of the PI-controller.

In Figure 11 and 12, the controller was turned on when the sustained oscillatory behavior in PSD distribution, measured as $\left(d_{50}\right)$, of the particles leaving the granulator was observed. Particularly, the controller was turned on at the maximum point of the cycle, at $t=20 \mathrm{~h}$ with $d_{\text {crush }}=1.3 \mathrm{~mm}$.

\section{Figure 11}

Control input and control output when the classical PIcontroller is used: controller turned on at the maximum point of the cycle

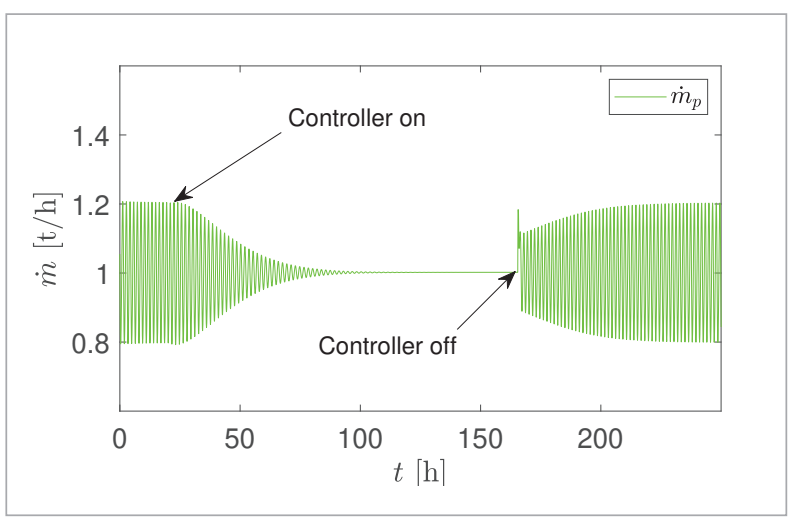

Figure 12

Product-sized mass flow when the classical PI-controller is used: controller turned on at the maximum point of the cycle

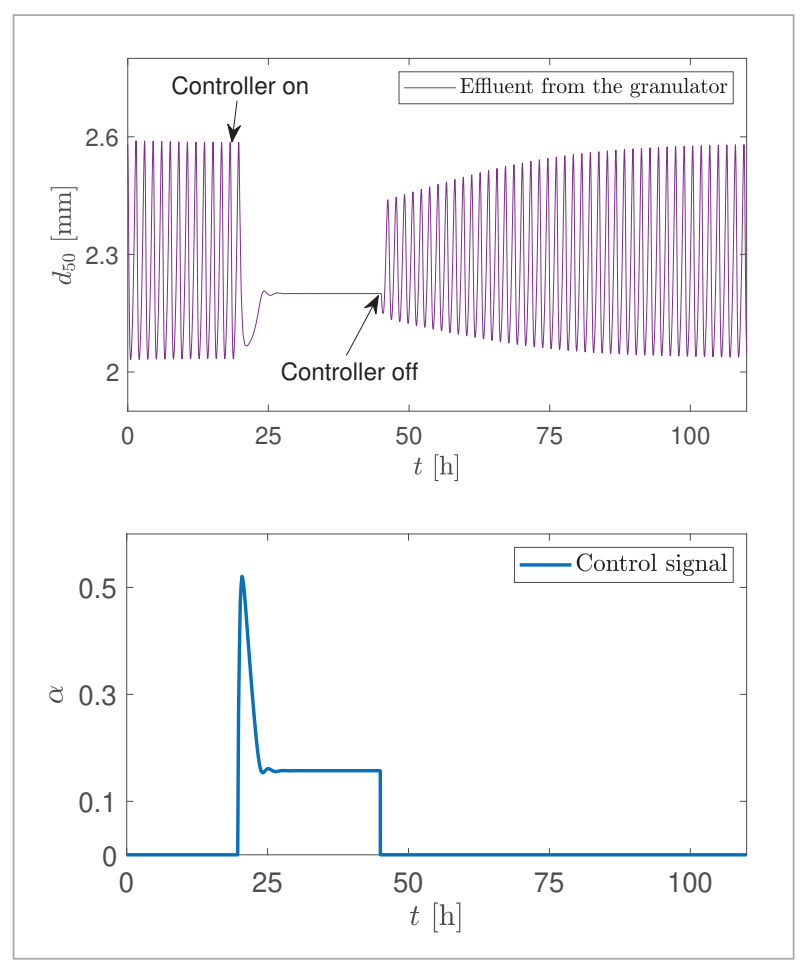

Simulation results using the classical PI-controller showed that it is possible to use a simple PI controller in order to eliminate the oscillatory behaviour in product quality (PSDs) and quantity (mass flow rate). When the PI controller was turned on, the oscillations were suppressed (damped) and the desired reference point was tracked. However, the convergence rate towards the operating point was relatively slow (it took more than $24 \mathrm{~h}$ of process simulation). This would limit the use of a such control strategy in a industrial-scale plant.

Simulations results when the double-loop control strategy was used are depicted in Figures 13 and 14. Similarly to simulations with the classical PI-controller, these simulations were also performed when the sustained oscillations in the granulation process were observed, i.e., at $d_{\text {crush }}=1.3$. For a fair comparison, in Figures 13 and 14 the controller was turned on at the same maximum point of the cycle, at $t=20$. With the double-loop control strategy the oscil- 


\section{Figure 13}

Control input and control output when the double-loop control strategy is used: controller turned on at the maximum point of the cycle [29]

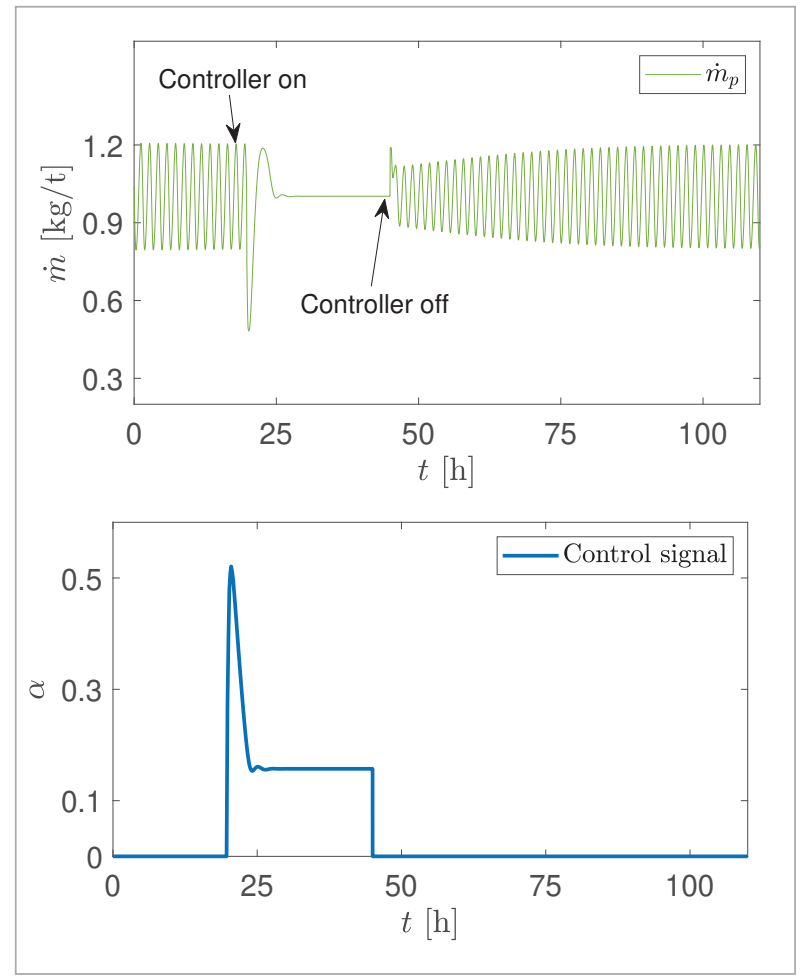

\section{Figure 14}

Product-sized mass flow when the double-loop control strategy is used: controller turned on at the maximum point of the cycle

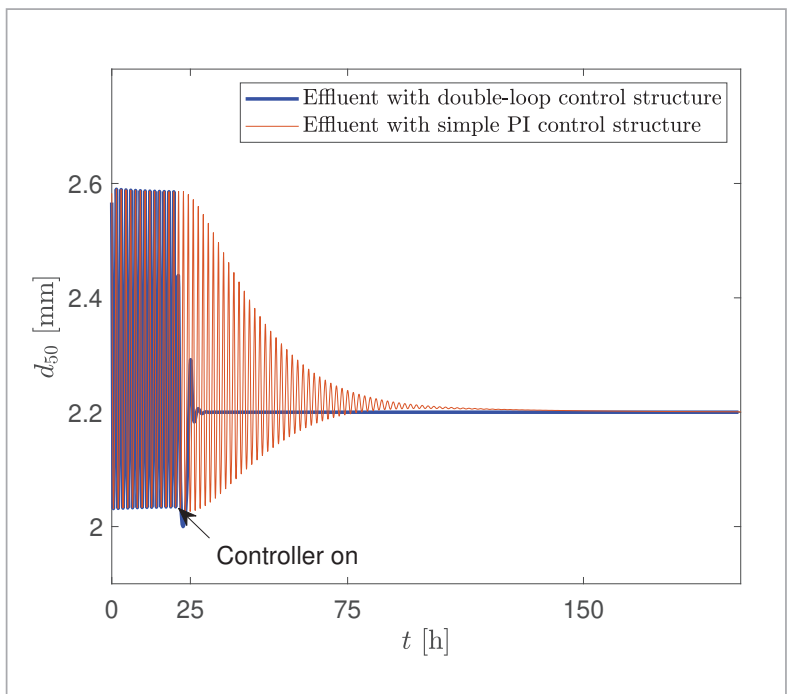

lations were quickly damped when the controller was turned on - a non-oscillating particle $d_{50}$ and mass flow rates of the effluent were obtained. It took only around $6 \mathrm{~h}$ to reach steady state value when the controller was turned on. Thus, with the double control strategy it is possible to reach significantly faster controller response compared to the classical PI-controller.

For further understanding of the controller behavior, the controller was turned on at the minimum point of the cycle (Figure 15). For fair comparison, identical controller parameters were used when controller was turned on at maximum and minimum cycle points. These simulations were performed both with the classical PI-controller and with the double-loop control strategy. In Figure 15, the response in the controlled variable for both control strategies are shown.

\section{Figure 15}

Classical PI-controller vs double-loop control strategy: controller turned on at the minimum point of the cycle

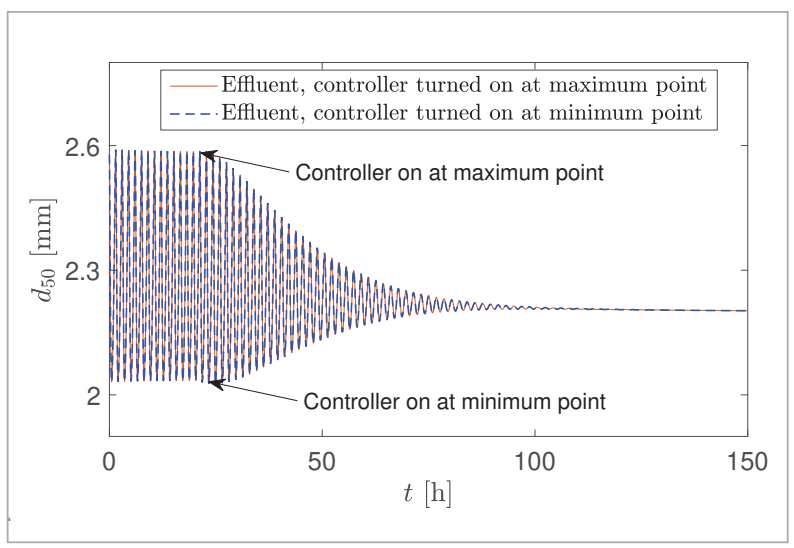

Similarly to the previous simulation results, significantly faster convergence rate towards the operating point is achieved when double-loop control strategy is used. As to the position in the cycle when the controller was turned on, no significant differences were seen for either control strategies, Figure 16 and 17. In the case of the double-loop control strategy, a slightly faster convergence rate towards the operating point is achieved when the controller is turned on at the maximum point of the cycle, $7 \mathrm{~h}$ vs $6 \mathrm{~h}$ with the minimum and maximum points respectively (Figure 17). To sum up, stabilization of the granulation process is achieved significantly faster with the 
double-loop control strategy than with the classical PI-controller.

For both control strategies, the point of the cycle at which the controller is turned on (maximum or minimum point of the cycle) does not significantly affect

\section{Figure 16}

Comparison of the control outputs for the classical PI-controller: controller turned on at minimum vs maximum point

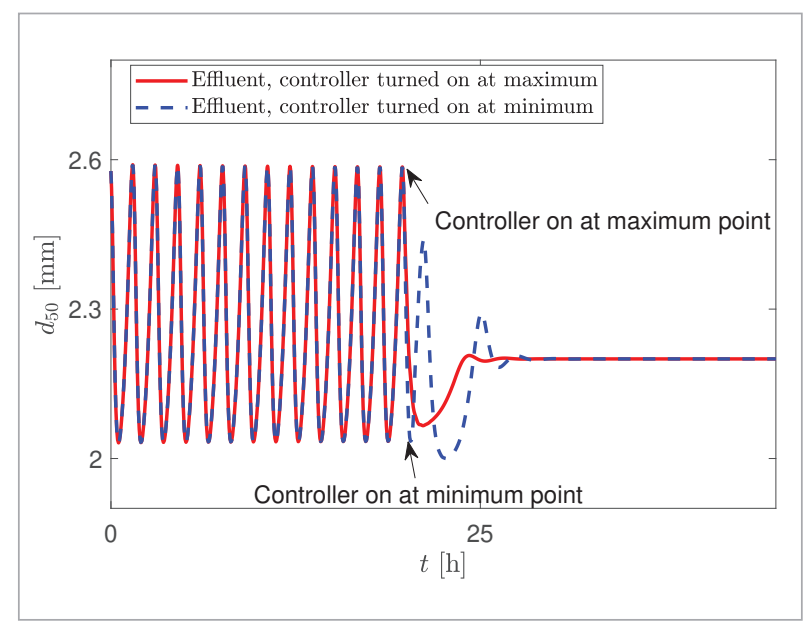

the closed loop response. This allows the operators to instantaneously turn on the controller without having to wait for several hours to reach either the maximum or minimum point of the cycle.

The double-loop control strategy is based on the well-established linear system theory which is already widely excepted in the process industry. This also allows for easier integration of this control strategy in the existing control system. Implementation requires to combine current on-line sensors for particle size distribution to provide an estimate of the median $d_{50}$. Online particle size analyzers are commercially available and often installed in granulation plants. Such devices can analyze several properties related to size and shape of particles, and the median $d_{50}$ is used as a measurement to a PID controller in the Process Control System. In addition, process must allow for direct manipulation (preferably automatic) of the discussed control input, the fraction of return flow. The double-loop control strategy uses a combination of a P controller and a PI controller, and the design of the inner loop controller provides flexibility in the sense that the operator can freely choose the desired damping ratio and calcu-

\section{Figure 17}

Disturbance rejection: Disturbances are applied at $t=20 \mathrm{~h}$ and $t=65 \mathrm{~h}$ (controller is turned on), and at $t=142 \mathrm{~h}$ (controller is turned off, $\left.u_{\text {manual }}=u_{\text {auto }}\right)$
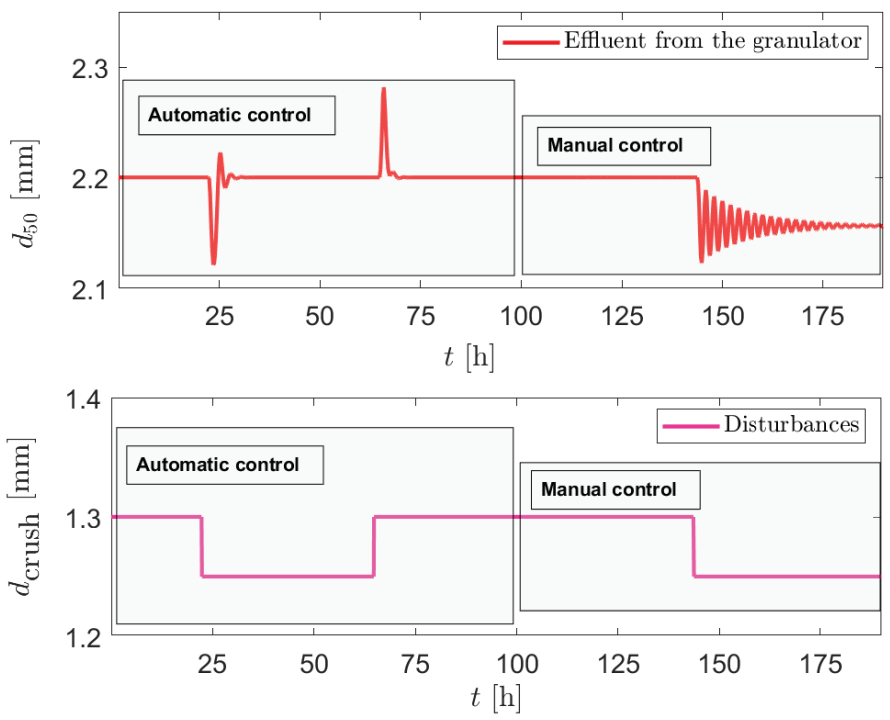
late the gain of the inner controller. The additional inner loop of the double control strategy does make the control system slightly complex compared to the classical PI controller. However, the advantage with the double-loop control strategy is that the oscillatory behaviour is suppressed significantly faster. This also means shorter transient periods in the plant and, hence, less production of the off-spec particles (over-sized and under-sized particles). Faster and tighter control of the product specification $\left(d_{50}\right.$ of the effluent from the granulator) leads to improved operation with further means improved profit. With the double-loop control strategy the particle mass flow rates also converges towards the operating point significantly faster compared to the classical PI controller. A stable mass flow rate also eliminates overloading or under loading of various equipment in the granulation loop.

Figure 17 shows that the double-loop control strategy is able to compensate for disturbances. Here, a crusher gap spacing is used as the disturbance. First, disturbances are applied when the controller is turned on. Second, the controller is turned off using the last value of the control input that is obtained while the controller was turned on. When the controller is turned off and the disturbance is applied to the system, the oscillatory behaviour in the particle $d_{50}$ reappears (Figure 17). Like the double-loop control strategy, the classical PI controller is able compensate for the disturbances, however, not illustrated in this paper for the sake of brevity.

\section{Appendix 1}

\begin{tabular}{c|l}
\hline Symbol & \multicolumn{1}{|c}{ Description } \\
\hline$B$ & birth rate due to particle agglomeration \\
\hline$D$ & death rate due to particle agglomeration \\
\hline$G$ & growth rate due to particle layering \\
\hline$H$ & Heaviside step function \\
\hline$K_{\mathrm{eff}}$ & separation efficiency of the screen \\
\hline$K_{\mathrm{c}}$ & proportional gain of the controller \\
\hline$T_{\mathrm{i}}$ & integral time constant \\
\hline$K_{\mathrm{p}}$ & process gain
\end{tabular}

\section{Conclusions}

The model presented in this paper was found to reproduce oscillatory behaviour of the granulation loop process seen on industrial-scale granulation plants. According to the performed bifurcation analysis, oscillatory behaviour in the particle $d_{50}$ occurs at crusher gap spacing $d_{\text {crush }}<1.4 \mathrm{~mm}$. Dynamic open loop simulations showed that it is possible to suppress oscillatory behavior of granulation loop process by recycling back some fraction of the product-sized particles.

Two feedback control strategies are proposed to suppress the oscillatory behavior of granulation loop process. Both control strategies utilize the most used control algorithm in the industry, namely the PI controller. In the first control strategy, a classical PI-controller is used. In the second - a double-loop control strategy with the two controllers, a P-controller and a PI-controller. Closed loop simulation results showed that both control strategies are able to suppress oscillatory behavior in PSD of the granules leaving the granulator. Different simulation scenarios are performed for understanding the controllers behaviors.

Simulation results showed that there is no significant difference in the dynamic response when the controller is turned on at the minimum point in the cycle or at the maximum point. The comparison between the two proposed control strategies shows that it is possible to achieve faster convergence rate towards the operating point when the double-loop control strategy is used. Thus, it can be advantageous to use the double-loop control strategy to make the granulation loop processes more steady to operate.

\begin{tabular}{c|l}
\hline $\begin{array}{c}\text { Symbol } \\
P(s)\end{array}$ & \multicolumn{1}{|c}{ Description } \\
\hline$d$ & transfer function \\
\hline$d_{50}$ & particle diameter \\
\hline$d_{\text {screen }}$ & mesh size of the screen \\
\hline$m$ & mass density function \\
\hline$\dot{m}$ & mass flow rate \\
\hline$t$ & time \\
\hline$v$ & velocity \\
\hline
\end{tabular}




\begin{tabular}{c|l} 
Symbol & \multicolumn{1}{|c}{ Description } \\
\hline$u$ & manipulatable variable \\
\hline$y$ & controlled variable \\
\hline$\alpha$ & valve opening \\
\hline$\beta$ & agglomeration rate (kernel) \\
\hline$\gamma$ & particle size distribution function \\
\hline$\rho$ & probability function of the screen \\
\hline$\sigma$ & particle density \\
\hline$\tau$ & standard deviation \\
\hline$\zeta$ & time delay \\
\hline$\lambda$ & damping factor \\
\hline$\omega$ & dimensionless term \\
\hline
\end{tabular}

\section{Acknowledgment}

The economic support from The Research Council of Norway and Yara Technology and Projects through project no. 26950\%/O20 'Exploiting multi-scale simu-

\section{References}

1. Bück, A., Dürr, R., Schmidt, M., Tsotsas, E. Model Predictive Control of Continuous Layering Granulation in Fluidised Beds with Internal Product Classification. Journal of Process Control, 2016, 45, 65-75. https://doi. org/10.1016/j.jprocont.2016.07.003

2. Bück, A., Palis, S., Tsotsas, E. Model-Based Control of Particle Properties in Fluidised Bed Spray Granulation. Powder Technology, 2015, 270, 575-583. https://doi. org/10.1016/j.powtec.2014.07.023

3. Cameron, I., Wang, F., Immanuel, C., Stepanek, F. Process Systems Modelling and Applications in Granulation: A Review. Chemical Engineering Science, 2005, 60(14), 3723-3750. https://doi.org/10.1016/j.ces.2005.02.004

4. Drechsler, J., Peglow, M., Heinrich, S., Ihlow, M., Mörl, L. Investigating the Dynamic Behaviour of Fluidized Bed Spray Granulation Processes Applying Numerical Simulation Tools. Chemical Engineering Science, 2005, 60(14), 3817-3833. https://doi.org/10.1016/j.ces.2005.02.010

5. Glaser, T., Sanders, C., Wang, F., Cameron, I., Litster, J., Poon, J. M.-H., Ramachandran, R., Immanuel, C., Doyle III, F. Model Predictive Control of Continu-

\begin{tabular}{c|l}
\hline $\begin{array}{c}\text { Subscript } \\
\text { crush }\end{array}$ & \multicolumn{1}{|c}{ crusher } \\
\hline $\mathrm{e}$ & effluent from the granulator \\
\hline $\mathrm{i}$ & influent to the granulator \\
\hline $\mathrm{o}$ & over-sized particles \\
\hline $\mathrm{p}$ & product-sized particles \\
\hline $\mathrm{sl}$ & slurry \\
\hline $\mathrm{u}$ & under-sized particles \\
\hline upp & upper screen \\
\hline low & lower screen \\
\hline$i$ & size class \\
\hline$z$ & compartment of the granulator \\
\hline
\end{tabular}

lation and control in developing next generation high efficiency fertilizer technologies (HEFTY)' is gratefully acknowledged.

ous Drum Granulation. Journal of Process Control, 2009, 19(4), 615-622. https://doi.org/10.1016/j.jprocont.2008.09.001

6. Heinrich, S., Peglow, M., Ihlow, M., Mörl, L. Particle Population Modeling in Fluidized Bed-Spray Granulation. Analysis of the Steady State and Unsteady Behavior. Powder Technology, 2003, 130(1-3), 154-161. https://doi.org/10.1016/S0032-5910(02)00259-0

7. Herce, C., Gil, A., Gil, M., Cortes, C. A Capetaguchi Combined Method to Optimize a NPK Fertilizer Plant Including Population Balance Modeling of Granulation-Drying Rotary Drum Reactor. In Computer Aided Chemical Engineering, 2017, 40, 49-54. https://doi. org/10.1016/B978-0-444-63965-3.50010-6

8. Iveson, S., Litster, J., Hapgood, K., Ennis, B. Nucleation, Growth and Breakage Phenomena in Agitated Wet Granulation Processes: A Review. Powder Technology, 2001, 117(1-2), 3-39. https://doi.org/10.1016/S00325910(01)00313-8

9. Koren, B. A Robust Upwind Discretization Method for Advection, Diffusion and Source terms. In Vreugdenhil, 
C. B., Koren, B. Numerical Methods for Advection-Diffusion Problems, Notes on Numerical Fluid Mechanics, 1993, 117-138.

10. Kumar, J. Numerical Approximations of Population Balance Equations in Particulate Systems. Ph.D. Thesis, Otto-von-Guericke-Universitat Magdeburg, Universitatsbibliothek, 2006.

11. Kumar, J., Peglow, M., Warnecke, G., Heinrich, S., Mörl, L. Improved Accuracy and Convergence of Discretized Population Balance for Aggregation: The Cell Average Technique. Chemical Engineering Science, 2006, 61(10), 3327-3342. https://doi.org/10.1016/j. ces.2005.12.014

12. Litster, J., Ennis, B. The Science and Engineering of Granulation Processes, Springer Science \& Business Media, 2004. https://doi.org/10.1007/978-94-0170546-2

13. Manum, H. Extensions of Skogestad's SIMC Tuning Rules to Oscillatory and Unstable Processes. NTNU Technical Report, 2005. Viewed 17 November 2020, http://folk.ntnu.no/skoge/diplom/prosjekt05/manum/ rapport.pdf

14. MATLAB. The MathWorks, Inc., Natick, Massachusetts, United States, 2019a.

15. Molerus, O., Hoffmann, H. Darstellung von Windsichtertrennkurven Durch ein Stochastisches Modell. Chemie Ingenieur Technik, 1969, 41(5-6), 340-344. https:// doi.org/10.1002/cite.330410523

16. Nandong, J. Double-Loop Control Structure for Oscillatory Systems: Improved PID Tuning via Multiscale Control Scheme. In 10th Asian Control Conference (ASCC), IEEE, 2015, 1-6. https://doi.org/10.1109/ ASCC.2015.7244476

17. Neugebauer, C., Bück, A., Kienle, A. Control of Particle Size and Porosity in Continuous Fluidized-Bed Layering Granulation Processes. Chemical Engineering and Technology, 2020,43(5), 813-8.https://doi.org/10.1002/ ceat.201900435

18. Neugebauer, C., Diez, E., Bück, A., Palis, S., Heinrich, S., Kienle, A. On the Dynamics and Control of Continuous Fluidized Bed Layering Granulation with Screen-MillCycle. Powder Technology, 2019, 354,765-78. https:// doi.org/10.1016/j.powtec.2019.05.030

19. Palis, S., Kienle, A. Stabilization of Continuous Fluidized Bed Spray Granulation - A Lyapunov Approach. IFAC Proceedings Volumes, 2010, 43(14), 1362-1367. https://doi.org/10.3182/20100901-3-IT-2016.00204
20. Palis, S., Kienle, A. Discrepancy Based Control of Continuous Fluidized Bed Spray Granulation with Internal Product Classification. IFAC Proceedings Volumes, 2012, 45(15), 756-761. https://doi. org/10.3182/20120710-4-SG-2026.00136

21. Palis, S., Kienle, A. Stabilization of Continuous Fluidized Bed Spray Granulation with External Product Classification. Chemical Engineering Science, 2012, 70,200-209. https://doi.org/10.1016/j.ces.2011.08.026

22. Park, J. H., Sung, S. W., Lee, I.-B. An Enhanced PID Control Strategy for Unstable Processes. Automatica, 1998, 34(6), 751-756. https://doi.org/10.1016/S00051098(97)00235-5

23. Radichkov, R., Müller, T., Kienle, A., Heinrich, S., Peglow, M., Mörl, L. A Numerical Bifurcation Analysis of Continuous Fluidized Bed Spray Granulator with External Product Classification. Chemical Engineering and Processing, 2006, 45,826-837. https://doi. org/10.1016/j.cep.2006.02.003

24. Ramachandran, R., Chaudhury, A. Model Based Design and Control of a Continuous Drum Granulation Process. Chemical Engineering Research and Design, 2012, 90(8), 1063-1073. https://doi.org/10.1016/j. cherd.2011.10.022

25. Ramachandran, R., Immanuel, C. D., Stepanek, F., Litster, J. D., and Doyle III, F. J. A Mechanistic Model for Breakage in Population Balances of Granulation: Theoretical Kernel Development and Experimental Validation. Chemical Engineering Research and Design, 2009, 87 (4), 598-614. https://doi.org/10.1016/j.cherd.2008.11.00'7

26. Ramkrishna, D. Population Balances: Theory and Applications to Particulate Systems in Engineering. Academic Press, 2000.

27. Skogestad, S. Simple Analytic Rules for Model Reduction and PID Controller Tuning. Journal of Process Control, 2003, 13(4), 291-309. https://doi.org/10.1016/ S0959-1524(02)00062-8

28. Valiulis, G., Simutis, R. Particle Growth Modelling and Simulation in Drum Granulator-Dryer. Information Technology and Control, 2009, 38(2), 147-152.

29. Vesjolaja, L., Glemmestad, B., Lie, B. Double-Loop Control Structure for Rotary Drum Granulation Loop. Processes, 2020, 8, 1423. https://doi.org/10.3390/ pr8111423

30. Vesjolaja, L., Glemmestad, B., Lie, B. Dynamic Model for Simulating Transient Behaviour of Rotary Drum Granulation Loop. Modeling, Identification and 
Control, 2020, 41(2), 65-777. https://doi.org/10.4173/ mic.2020.2.3

31. Vesjolaja, L., Glemmestad, B.,Lie, B. Population Balance Modelling for Fertilizer Granulation Process. Linköping Electronic Conference Proceedings, 2018, 153, 95-102. https://doi.org/10.3384/ecp1815395

32. Wang, F., Cameron, I. A Multi-Form Modelling Ap- proach to the Dynamics and Control of Drum Granulation Processes. Powder Technology, 2007, 179(1-2), 2-11. https://doi.org/10.1016/j.powtec.2006.11.003

33. Wang, F., Ge, X., Balliu, N., Cameron, I. Optimal Control and Operation of Drum Granulation Processes. Chemical Engineering Science, 2006, 61(1), 257-267. https:// doi.org/10.1016/j.ces.2004.11.067 